\title{
Attenuation correction for myocardial perfusion SPECT imaging: still a controversial issue
}

\author{
Alberto Cuocolo \\ Received: 28 July 2011 / Accepted: 29 July 2011 /Published online: 27 August 2011 \\ (C) Springer-Verlag 2011
}

The use of attenuation correction for cardiac single photon emission computed tomography (SPECT) myocardial perfusion imaging has been discussed from the beginning. The value of attenuation correction is easy to accept, and it is generally recognized that it is important to discern if a tracer deficit is due to diminished myocardial perfusion or if it is an attenuation or motion artefact. An additional important problem is to establish whether the defect is really reversible or represents a result of scatter and interference from extracardiac tracer activity. It seems conceivable that attenuation correction should at least improve the problems related with an attenuation artefact. There are also additional potential advantages of attenuation correction, which have been outlined in many studies. However, the question of whether attenuation correction is needed is still unsolved and it has been debated for a long time.

From one side, attenuation correction is a comprehensive method to improve the accuracy of how the true tracer concentration is extracted from the images [1, 2]. Several clinical trials have shown that attenuation correction improves the diagnostic accuracy of myocardial perfusion imaging for detecting coronary artery disease [3-13]. Practically every clinical trial that has been performed to determine the diagnostic capability of attenuation-corrected SPECT myocardial perfusion imaging has resulted in improved diagnostic accuracy over uncorrected imaging. This is true whether the interpretation has been performed

A. Cuocolo $(\bowtie)$

Department of Biomorphological and Functional Sciences,

University Federico II,

Naples, Italy

e-mail: cuocolo@unina.it with visual or quantitative analysis [3]. Some studies also showed that attenuation correction is incremental to improvements resulting from gated SPECT imaging [4]. The improved diagnostic performance is more evident in a heavier patient population $[3,4]$. The most common finding in these trials is that, compared with non-attenuationcorrected SPECT, attenuation correction significantly improves the normalcy rate and the specificity for detecting coronary artery disease. These clinical trials have persuaded the professional societies to endorse the clinical use of attenuation correction in myocardial perfusion SPECT imaging. On the basis of the available clinical evidence and the rapid development of attenuation correction technology, the American Society of Nuclear Cardiology and the Society of Nuclear Medicine recommended that providers consider the addition of hardware and software that have undergone clinical validation and include appropriate quality control tools to perform nonuniform attenuation correction [14]. Currently, it is suggested that both non-corrected and corrected image sets be reviewed and integrated into the final report. However, as the reader gains the appropriate experience and confidence in correction methodology, only the corrected images may be necessary, as is the standard in positron emission tomography. On the basis of current information and the rate of technology improvement, the American Society of Nuclear Cardiology and the Society of Nuclear Medicine believe that attenuation correction should be regarded as a rapidly evolving standard for SPECT myocardial perfusion imaging. Therefore, it is their recommendation that the adjunctive technique of attenuation correction has become a method for which the weight of evidence and opinion is in favour of its usefulness [14]. In addition, attenuation correction is an essential requirement for accurately extracting quantitative parameters 
from all types of cardiac radionuclide distributions that should play an essential role in establishing cardiac SPECT for flow, metabolic, innervation and molecular imaging [1].

Currently the most widely used attenuation correction instrumentation utilizes single-slice computed X-ray tomography (CT) for acquisition of transmission maps [15]. However, several reports on the use of attenuation correction in myocardial SPECT imaging showed discordant results and, therefore, its utility in routine clinical practice is still debated [1,2]. The current study by Genovesi et al. [16] sought to evaluate the effect of CTbased attenuation correction and of gated acquisition on the interpretation of myocardial SPECT in a multicentre investigation involving patients with known coronary anatomy. According to their results, CT-based nonuniform attenuation correction of ${ }^{99 \mathrm{~m}} \mathrm{Tc}$-tetrofosmin gated SPECT fast imaging consistently improved specificity without affecting sensitivity only in the evaluation of the inferior wall in overweight men. In the other evaluable subgroups of patients specificity was not significantly affected while sensitivity was frequently reduced. Since the number of overweight women was too small to perform statistical analysis, conclusions on this specific subgroup could not be obtained. In addition, as the authors underlined, the routine application of attenuation correction brings in extra radiation exposure to patients. Although these dose values are relatively low, this does not justify the extensive use of attenuation correction, especially in patients that will not benefit from it. In a previous study, Wolak et al. [17] compared quantitative attenuation-corrected data specifically in a female population. The quantitative analysis used in that investigation avoided any kind of bias that might be possible with visual analysis. Their results showed that there is no measurable difference in the degree of automation and in the diagnostic performance when attenuation-corrected and non-corrected data for women are compared. In particular, attenuation correction was associated with false-positive results in the left anterior descending artery territory, whereas non-correction was associated with false-positive results in the right coronary artery territory [17]. As reported by these authors, the apparent lack of advantage of attenuation correction over non-correction in the diagnostic performance of quantification can have many explanations. The improved diagnostic performance of attenuation correction in the large majority of previous studies was attributed mainly to improvement in the normalcy rate and specificity for detecting coronary artery disease. However, because of the apical thinning and truncation artefacts induced by attenuation correction, this procedure might create false-positive perfusion defects in a normal myocardium, leading to a reduced specificity $[18$, 19]. Visual analysis might be able to circumvent this problem, because all of the reported studies used both non-corrected and attenuation-corrected data in the final visual analysis. Interestingly, the relative incidence of apical thinning was found to be more than twice as high for women as for men [19], and therefore may represent a relatively larger problem in women than in the general population. Furthermore, the low specificity of most quantitative programs with non-corrected data reported in previous studies was often associated with older algorithms, which are limited by low specificity [20]. More recent algorithms developed by different groups achieved higher specificity but did not support the hypothesis of a significant measurable advantage of attenuation correction [21]. Therefore, it is possible that the incremental diagnostic benefit of attenuation correction is inversely related to the sophistication of the specific quantitative algorithm used to measure it. The results of the study by Genovesi et al. [16] suggest that in clinical practice the use of attenuation correction should be limited to male patients with a body mass index higher than 27. In the other subgroups of patients standard gated SPECT should be preferred. Furthermore "dosimetry-adapted" softwares are needed to avoid unnecessary CT scans in order to further reduce the total effective dose to the patient.

As stressed by Germano et al. [2] the great variety of available attenuation correction hardware and software flavours, the historical pattern of commercial release of insufficiently validated attenuation correction implementations and the increasingly clouded health care reimbursement horizon may have created an environment where the envisioned users of the technology have been desensitized and discouraged from expecting it to ever come to fruition in a standardized, validated and cost-effective form. It has been reported that the entire base of SPECT cameras has only 5\% of systems with attenuation correction [22]. Perhaps the reports of attenuation correction decline are overstated, but it seems that the future of attenuation correction has not yet been clearly defined [2]. Most clinicians would agree that achieving the ideal of attenuation correction has been more complex than was generally imagined, and the way has been lined with solutions that were disappointing. The correction for "attenuation" turns out to be not entirely correct, and although attenuation artefacts are diminished, some new artefacts can be created from the attenuation correction process. From the viewpoint of a practising clinician, attenuation correction adds complexity and cost and replaces the old familiar artefacts with some new ones that are strange and unpredictable. Moreover, it is also important to underline that this procedure is not reimbursed and that reimbursement clearly represents a key issue. The engineering required to render attenuation correction simple and robust for general clinical applications is difficult and expensive. Without reimbursement, there is little to support such massive engineering 
refinement. It is also argued that until attenuation correction becomes essential and ubiquitous for general clinical use, there will be no incentive to reimburse. All these challenges, including those reported by Genovesi et al. [16], have slowed the wider clinical use of attenuation correction techniques for SPECT myocardial perfusion imaging.

\section{References}

1. Garcia EV. SPECT attenuation correction: an essential tool to realize nuclear cardiology's manifest destiny. J Nucl Cardiol 2007;14:16-24.

2. Germano G, Slomka PJ, Berman DS. Attenuation correction in cardiac SPECT: the boy who cried wolf? J Nucl Cardiol 2007;14:25-35.

3. Grossman GB, Garcia EV, Bateman TM, Heller GV, Johnson LL, Folks RD, et al. Quantitative Tc-99m sestamibi attenuation-corrected SPECT: development and multicenter trial validation of myocardial perfusion stress gender-independent normal database in an obese population. J Nucl Cardiol 2004;11:263-72.

4. Thompson RC, Heller GV, Johnson LJ, Case JA, Cullom SJ, Garcia EV, et al. Value of attenuation correction on ECG-gated myocardial perfusion imaging related to body mass index. J Nucl Cardiol 2005;12:195-202.

5. Ficaro EP, Fessler JA, Shreve PD, Kritzman JN, Rose PA, Corbett JR. Simultaneous transmission/emission myocardial perfusion tomography. Diagnostic accuracy of attenuation-corrected 99mTc-sestamibi single-photon emission computed tomography. Circulation 1996;93:463-73.

6. Gallowitsch HJ, Sykora J, Mikosch P, Kresnik E, Unterweger O, Molnar M, et al. Attenuation-corrected thallium-201 single-photon emission tomography using a gadolinium-153 moving line source: clinical value and the impact of attenuation correction on the extent and severity of perfusion abnormalities. Eur J Nucl Med 1998;25:220-8.

7. Hendel RC, Berman DS, Cullom SJ, Follansbee W, Heller GV, $\mathrm{Kiat} \mathrm{H}$, et al. Multicenter clinical trial to evaluate the efficacy of correction for photon attenuation and scatter in SPECT myocardial perfusion imaging. Circulation 1999;99:2742-9.

8. Links JM, Becker LC, Rigo P, Taillefer R, Hanelin L, Anstett F, et al. Combined corrections for attenuation, depth-dependent blur, and motion in cardiac SPECT: a multicenter trial. J Nucl Cardiol 2000;7:414-25.

9. Shotwell M, Singh BM, Fortman C, Bauman BD, Lukes J, Gerson MC. Improved coronary disease detection with quantitative attenuation-corrected Tl-201 images. J Nucl Cardiol 2002;9:52-62.

10. Slart RH, Que TH, van Veldhuisen DJ, Poot L, Blanksma PK, Piers DA, et al. Effect of attenuation correction on the interpre- tation of $99 \mathrm{mTc}-$ sestamibi myocardial perfusion scintigraphy: the impact of 1 year's experience. Eur J Nucl Med Mol Imaging 2003;30:1505-9.

11. Banzo I, Pena FJ, Allende RH, Quirce R, Carrill JM. Prospective clinical comparison of non-corrected and attenuation- and scatter-corrected myocardial perfusion SPECT in patients with suspicion of coronary artery disease. Nucl Med Commun 2003;24:995-1002.

12. Masood Y, Liu YH, DePuey G, Taillefer R, Araujo LI, Allen S, et al. Clinical validation of SPECT attenuation correction using $\mathrm{x}$-ray computed tomography-derived attenuation maps: multicenter clinical trial with angiographic correlation. J Nucl Cardiol 2005; $12: 676-86$

13. Utsunomiya D, Tomiguchi S, Shiraishi S, Yamada K, Honda T, Kawanaka $\mathrm{K}$, et al. Initial experience with X-ray CT based attenuation correction in myocardial perfusion SPECT imaging using a combined SPECT/CT system. Ann Nucl Med 2005;19:485-9.

14. Hendel RC, Corbett JR, Cullom SJ, DePuey EG, Garcia EV, Bateman TM. The value and practice of attenuation correction for myocardial perfusion SPECT imaging: a joint position statement from the American Society of Nuclear Cardiology and the Society of Nuclear Medicine. J Nucl Cardiol 2002;9:135-43.

15. Patton JA, Townsend DW, Hutton BF. Hybrid imaging technology: from dreams and vision to clinical devices. Semin Nucl Med 2009;39:247-63.

16. Genovesi D, Giorgetti A, Gimelli A, Kusch A, D'Aragona Tagliavia I, Casagranda M, et al. Impact of attenuation correction and gated acquisition in SPECT myocardial perfusion imaging: results of the multicentre SPAG (SPECT Attenuation Correction vs Gated) study. Eur J Nucl Med Mol Imaging 2011. doi:10.1007/ s00259-011-1855-4.

17. Wolak A, Slomka PJ, Fish MB, Lorenzo S, Berman DS, Germano G. Quantitative diagnostic performance of myocardial perfusion SPECT with attenuation correction in women. J Nucl Med 2008;49:915-22.

18. Case J, Cullom S, Bateman TM. Myocardial perfusion singlephoton emission computed tomography attenuation correction. 3rd ed. New York: Oxford University Press; 2003.

19. Links JM, Becker LC, Anstett F. Clinical significance of apical thinning after attenuation correction. J Nucl Cardiol 2004;11:2631.

20. Van Train KF, Garcia EV, Maddahi J, Areeda J, Cooke CD, Kiat H, et al. Multicenter trial validation for quantitative analysis of sameday rest-stress technetium-99m-sestamibi myocardial tomograms. J Nucl Med 1994;35:609-18.

21. Slomka PJ, Fish MB, Lorenzo S, Nishina H, Gerlach J, Berman DS, et al. Simplified normal limits and automated quantitative assessment for attenuation-corrected myocardial perfusion SPECT. J Nucl Cardiol 2006;13:642-51.

22. Hendel RC. Attenuation correction: eternal dilemma or real improvement? Q J Nucl Med Mol Imaging 2005;49:30-42. 